\title{
METASTATIC BREAST CARCINOMA MASQUERADING AS NON-RESOLVING PNEUMONIA
}

Deepak Rosha ${ }^{1}$, Trilok Chand ${ }^{2}$, Parminder B. Singh ${ }^{3}$

\section{HOW TO CITE THIS ARTICLE:}

Deepak Rosha, Trilok Chand, Parminder B. Singh. "Metastatic Breast Carcinoma Masquerading as NonResolving Pneumonia". Journal of Evolution of Medical and Dental Sciences 2014; Vol. 3, Issue 33, August 07; Page: 8924-8928, DOI: $10.14260 /$ jemds/2014/3145

ABSTRACT: Non Resolving pneumonias always present a difficult problem. Sometimes despite every effort, the diagnosis is not obvious. We report a case of ductal carcinoma of breast presenting as nonresolving pneumonia in a female patient with dry cough and bilateral crackles on physical examination. An initial CT Thorax showed pneumonic patches in the lingula and middle lobe. There was also mediastinal lymphadenopathy. She was evaluated by bronchoscopic procedures which were inconclusive except for chronic inflammation seen on trans-bronchial lung biopsy. A subsequent PETCT of the chest revealed patchy FDG avid sub-segmental collapse, consolidation with air bronchogram in lingular segment. There were also bilateralpulmonary few sub-centimeter nodular lesions andleft pleural thickening. However the primary breast lesion was not FDG avid. The diagnosis was made by lymph node biopsy of recently appearing supra-clavicular lymph node. The primary lesion was detected by clinical breast examination and confirmed by USG guided FNAC.

KEYWORDS: Non-resolving pneumonia, Ductal carcinoma breast, Metastatic lesions.

INTRODUCTION: Metastatic ductal carcinoma of breast presenting as non-resolving pneumonia is a rare presentation. Non-resolving pneumonia is commonly defined as the presence of focal infiltrates associated with symptoms of acute pulmonary infection and lack of clinical improvement or lack of resolution of infiltrates within 12 weeks despite a minimum of 10 days of antibiotic therapy.[1,2,3]

Sometimes delayed resolution of pneumonia is due to bronchogenic carcinoma with endobronchial occlusion, however adenocarcinoma in situ and primary pulmonary lymphomas or lymphoproliferative disorders, occasionally present with focal, alveolar opacities. Other malignancies rarely present as non-resolving pneumonia. ${ }^{[4,5]}$

CASE REPORT: A 56 years female presented with complaints of dry cough, decreased appetite and weight loss of two months duration. The patient had received multiple antibiotics with persistence of symptoms. The chest examination revealed bilateral basal crackles. The rest of clinical examination was unremarkable.

On investigation hematological parameters, liver and renal function test were within normal limits. The chest X-ray showed left upper zone and bilateral lower zone infiltrates and left parahilar opacity (Fig. 1). CECT Chest revealed few small $(<1 \mathrm{~cm})$ multiple nodular lesions bilaterally in subpleural as well as peribronchovacular distribution. There were fibrotic bands and thickened interlobular septae present in both lung fields.

There was also left pleural thickening, along with minimal left pleural effusion. Mediastinal lymphadenopathy in multiple lymph node stations was also seen with calcification in multiple lymph nodes. In addition there were areas of consolidation with accompanying air bronchograms seen in 
lingula and right middle lobe displayed scattered patchy shadows (Fig 2). Significantly no breast lesion was evident on CT Thorax.

A tuberculin test was negative and serum angiotensin converting enzyme (ACE) was not raised. Tests for auto-immune disorders, anti-nuclear antibodies, $\mathrm{P}$ and C-ANCA, and rheumatoid factor were negative. A bronchoscopy was done. The BAL AFB stain, fungal stain, gram stain were negative and AFB, aerobic and fungal culture showed no growth. TBNA of mediastinal lymph node was inconclusive. TBLB showed features of chronic inflammation, but no granulomas, or malignancy. Empirical ATT was started.

A Follow up CXR done two months later, showed slight improvement. The patient also reported decrease in cough and improvement in symptoms so the ATT was continued.

The patient received five months of ATT, but then complained of increase in cough. The CXR revealed essentially no change. On examination she had now a palpable left supraclavicular lymph node. A PET- CT of whole body was done which showed patchy FDG avid sub-segmental collapse consolidation with air bronchogramin lingula and right middle lobe with bilateral pulmonary nodular lesions and nodular left pleural thickening (Fig. 3).

There was FDG avid cervical, supraclavicular, mediastinal and abdominal lymphadenopathy. There were also mild FDG avid subtle lytic areas with sclerotic foci involving multiple cervical, dorsal and lumbar vertebrae. A left supraclavicular lymph node biopsy was done, which showed metastatic carcinoma with mucinous differentiation, the tumor cells were positive for estrogen receptors and origin of metastasis from breast was the most likely possibility. An ultrasound breast revealed evidence of spiculated ill-defined mass lesion in right upper quadrant measuring 2.1 x 1.8 $\mathrm{cm}$.

FNAC of the breast lesion was done which showed smears of scanty to moderate cellularity. Smears were composed of few clusters and singly dispersed malignant duct epithelial cells with hyperchromatic, pleomorphic nuclei, small distinct nucleoli and moderate amount of cytoplasm consistent with ductal carcinoma. The patient was referred to oncologist. With appropriate treatment for carcinoma breast the cough disappeared and appetite improved. There was also significant resolution of pneumonic patches.

DISCUSSION: Invasive ductal carcinoma is one of the major types of breast cancer, accounting for nearly $70 \%$ to $80 \%$ of presentation. Breast carcinoma metastasizes to distant sites by way of the angiolymphatic system. The first lymph nodes to be seeded with tumor cells are the axillary nodes, followed by the supraclavicular nodes and the mammary nodes.

Distant sites of metastases include the skeletal system, lungs and pleura, liver, ovaries, adrenal glands, and central nervous system including the leptomeninges and eyes. Four negatives of ER, PR, Her2/neu, and P53 have a tendency to initially metastasize to lungs.[6,7,8]

Breast cancer is the most common malignancy in women. The lung is a common site of metastasis and the only site in $21 \%$ of the patients.[9,10]In a study done by Kreisman $\mathrm{H}$ et al, lymphangitic carcinoma was the most common pattern of pulmonary parenchymal metastasis.

Multiple nodules occurred almost four times as often as solitary nodules. [11]However such kind of pneumonic patches as seen in our case suggesting an infective lesion are not described in the context of carcinoma breast. 


\section{CASE REPORT}

Breast examination remains a very critical part of clinical examination which is often not performed. Even so a mammography or USG of breast may have revealed the diagnosis of this patient and saved time as well as unnecessary exposure to ATT. An interesting feature of the case was that the primary breast lesion was not FDG avid. The axillary nodes were not palpable and also not FDG avid. It was also unusual to have the subsequent enlargement of a supraclavicular node without enlargement of lymph nodes more directly draining the primary lesion.

CONCLUSION: In conclusion this case emphasizes the importance of thorough evaluation of every case of non-resolving pneumonia, so that an occult malignancy is not missed. Malignancy remains a common differential diagnosis in patients having non-resolving pneumonia.

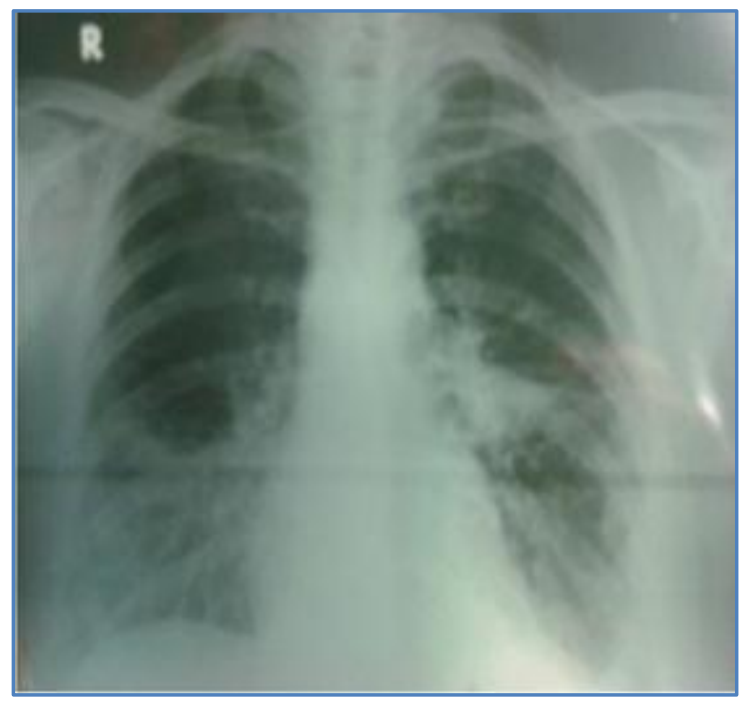

Fig.1: CXR showing patchy pneumonia in right middle lobe and lingual

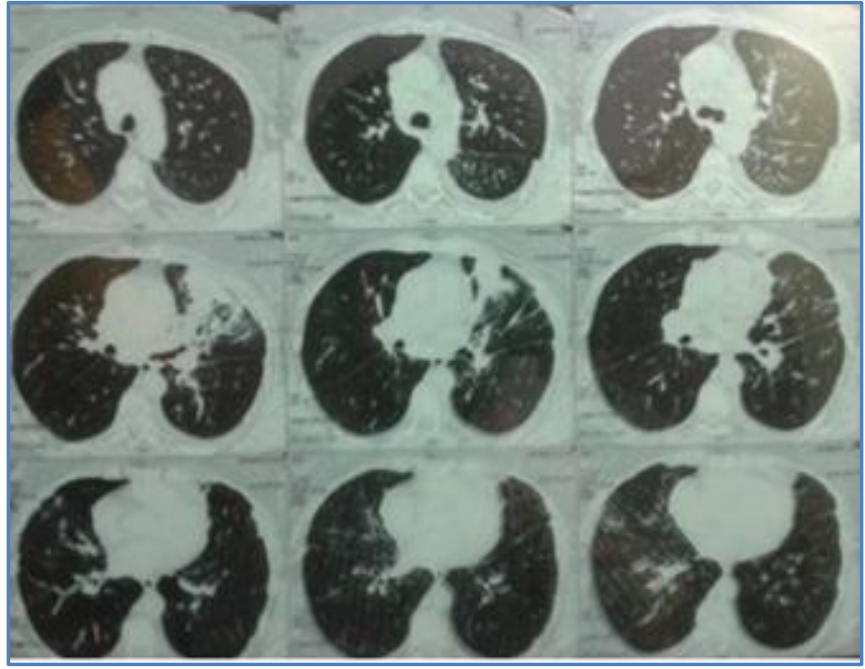

Fig. 2: CECT-Chest showing dense pneumonia with air bronchograms inlingula, and patchy shadows in right middle lobe 


\section{CASE REPORT}

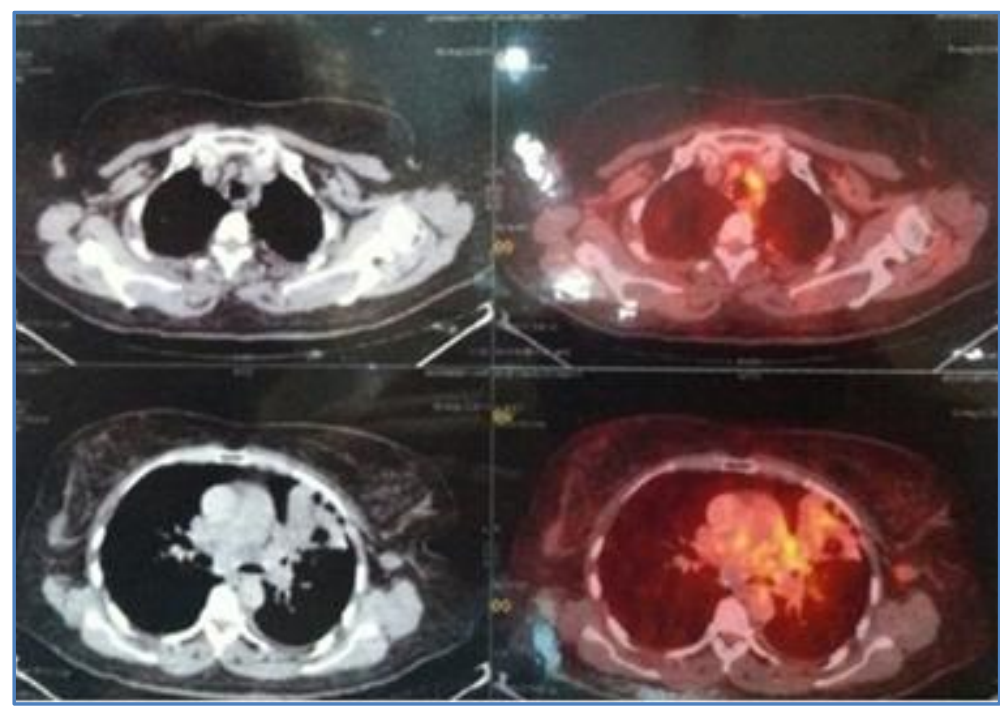

Fig. 3: PET- CT showing patchy FDG uptake in lingual

\section{REFERENCES:}

1. Mason RJ, Murray JF, Broaddus VC, Nadel JA. Pyogenic bacterial pneumonia and lung abscess. in: Murray and Nadel's textbook of respiratory medicine, Vol.1, 5th ed. Philadelphia; 201:699-40.

2. El Solh AA, Aquilina AT, Gunen H, Ramadan F. Radiographic resolution of community - acquired bacterial pneumonia in the elderly. J Am Geriatr Soc 2004; 52(2):224-29.

3. Weyers CM, Leeper KV. Non-resolving pneumonia. Clin Chest Med 2005; 26(1):143-58.

4. Barkley JE, Green MR. Bronchioloalveolar carcinoma. J Clin Oncol 1996; 14:2377-86.

5. Colby TV, Carrington CB. Pulmonary lymphomas. Current concepts. Hum Pathol 1983; 14:88487.

6. Kamby C, Andersen J, Ejlertsen B, Birkler NE, Rytter L, Zedeler K, et al. Pattern of spread and progression in relation to the characteristics of the primary tumor in human breast cancer. Acta Oncol 1991; 30:301-8.

7. Jain S, Fisher C, Smith P, Millis RR, Rubens RD. Patterns of metastatic breast cancer in relation to histological type. Eur J Cancer 1993; 15:2155-57.

8. Tomin R, Donegan WL. Screening for recurrent breast cancer-its effectiveness and prognostic value. J Clin Oncol 1987; 5:62-7.

9. Weiss L, Gilbert HA: Patterns of pulmonary metastases, in Weiss L, Gilbert HA (eds): Pulmonary Metastasis. Boston, GK Hall and Co, 1978:100-10.

10. Hagemeister FB, Buzdar AU, Luna MA, Blumenschein GR. Causes of death in breast cancer: a clinicopathologic study. Cancer 1980; 46:162-7.

11. Kreisman H, Wolkove N, Finkelstein H S, Carolcohen, Margolese R, Frank H.Breast cancer and thoracic metastases: review of 119 patients. Thorax 1983; 38:175-79. 


\section{CASE REPORT}

\section{AUTHORS:}

1. Deepak Rosha

2. Trilok Chand

3. Parminder B. Singh

\section{PARTICULARS OF CONTRIBUTORS:}

1. Senior Consultant, Department of Respiratory, Critical Care and Sleep Medicine, Indraprastha Apollo Hospitals, New Delhi, India.

2. Attending Consultant, Department of Respiratory, Critical Care and Sleep Medicine, Indraprastha Apollo Hospitals, New Delhi, India.

3. Registrar, Department of Respiratory, Critical Care and Sleep Medicine, Indraprastha Apollo Hospitals, New Delhi, India.

\section{NAME ADDRESS EMAIL ID OF THE}

\section{CORRESPONDING AUTHOR:}

Dr. Trilok Chand,

B-241, Sarita Vihar,

New Delhi-110076,

India.

Email: rao272@gmail.com

Date of Submission: 18/07/2014.

Date of Peer Review: 19/07/2014.

Date of Acceptance: 30/07/2014.

Date of Publishing: 06/08/2014. 